\title{
Improved Hydrodynamic Performance of Stepped-Slope floating Breakwater using Submerged Horizontal Plate
}

\begin{abstract}
E. Tolba ${ }^{1 / E}$. Galal ${ }^{2} / A$. Abou seeda ${ }^{3}$
\section{ABSTRACT}

Recently, the investigations on the hydrodynamic performance of floating breakwaters have great interest to researchers because of its high advantages in protecting marinas and small craft harbors. For the purpose of improving the hydrodynamic performance of the stepped-slope floating breakwater type, this research had been carried out as an approach to investigate experimentally the resulting hydrodynamic performance of a composite pile supported breakwater consists of a stepped-slope floating body moving only in heave motion overlying a fixed submerged horizontal plate in a constant water depth, $d=30 \mathrm{~cm}$. The transmission, reflection and losses coefficients had been determined individually for each of the stepped-slope floating, fixed submerged horizontal plate and the composite breakwaters. The results showed that the performance of the stepped-slope floating breakwater tested using different relative draft values, $D_{f} / d$, of the floating body had been significantly improved and achieved extra wave damping by reducing the transmission coefficients by a ratio range between $23 \%$ to $52 \%$, when using the submerged fixed horizontal plate underneath the floating body, with definite improvement value depends on plate relative submergence depth, $d_{p} / d$ and plate relative thickness, $t_{p} / d$. For a composite breakwater using two relative submergence depths of the plate, it had been found that a significant higher wave attenuation takes place with a configuration using $d_{p} / d=0.33$ than that obtained when using $d_{p} / d=0.50$. Furthermore, plate relative thickness achieved wave attenuation improvement for the composite breakwater especially for the configuration using $d_{p} / d=0.33$ and $t_{p} / d=4 \%$.
\end{abstract}

Keywords: Composite, Stepped, Floating Breakwater, Submerged plate, Transmission.

\section{INTRODUCTION}

Floating breakwaters are often used for temporary and permanent wave attenuation purposes. The temporary use of floating breakwaters is mostly happened during the executions of coastal projects for creating calm water area protecting the work site from waves attack. The permanent use of floating breakwaters is mainly applied for protecting marinas and small craft harbors. The advantages of floating breakwaters for both temporary and permanent usages are that; providing acceptable wave attenuation when exposed to moderate wave climate, allowing for water circulation and the sediment transport process. On the other hand, the floating breakwaters have disadvantages to withstand the energy of long waves.

The different types and shapes of floating breakwaters had been widely investigated during the past decades. The investigations on floating breakwaters had been initiated studying the performance of different floating configurations moored using flexible mooring lines.

${ }^{1}$ Associate professor, Faculty of Engineering, Port Said University, Egypt, Email: tolba_1966@yahoo.com

2 Assistant Professor, Faculty of Engineering, Port Said University, Egypt, Email: saydod@gmail.com ${ }^{3}$ Administrator, Civil Engineering, Higher institute of engineering and technology in New Damietta, Egypt,Email: eng_abouseeda@yahoo.com.
Tolba [19], studied experimentally the performance of floating breakwaters when supported using vertical piles allowing the structure to move only in heave with limited roll motions.

A lot of experimental and numerical researches after Tolba [19], had been carried out for pile supported floating breakwaters; Drieman [4], Fousert [5], Koftis. T., and Prinos. P. [8], Koftis et al [9], Koutandos et al [10], Koutandos et al [11], Koutandos et al [12], Koutandos, E. and Prinos. P [13] and Merlevede [15].

Furthermore, the fixed submerged horizontal plate acting as wave attenuator had been also widely investigated Aghili et al [1], Dauer [3], Graw [6], Patarapanich, M. and Cheong, H., [16], Tolba and Balah [20]. The hydrodynamic behaviors of a floating breakwater consisting of a rectangular pontoon with attached horizontal plates are studied numerically (Chen, et al., [2]) in which the fluid motion is idealized as twodimensional linear potential flow. The motions of the floating breakwater are assumed to be two-dimensional in sway, heave, and roll.

Recently, the stepped-slope floating breakwaters system is a point of interest for researchers. Teh [17], Teh and Ismail [18] investigated experimentally three types of model, namely the single-row, double-row and triple-row stepped-slope floating breakwaters, each test model was cross-moored to the bottom of the flume by four nylon ropes allowing the model to move in heave, sway and roll motions. 

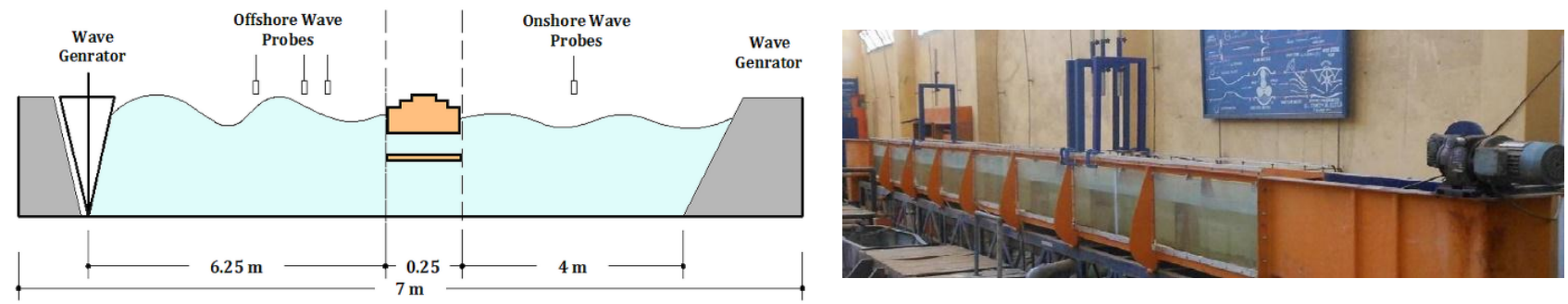

Figure 1: The overall view of the wave flume; the wave tank (left panel) and the experimental equipment (right panel).

Heng [7] studied the performance of step-float breakwaters using, chain mooring system, restrained system and pile-supported system moving in heave with limited roll motion, on various system arrangements and found that with 3-row, $G=b$ and $\mathrm{G}=2 \mathrm{~b}$ arrangements are capable to attenuate waves up to $80 \%$ of the incident wave height for wave period of less than 1.33 seconds. Kolahdoozan, et al., [14] studied the effect of step addition in longitudinal section of $\pi$-shaped FBs moving only in heave motion experimentally and using Support Vector Machine (SVM) method.

The main objective of this work is to investigate experimentally the hydrodynamic performance and improvements happen to the stepped-slope floating breakwater, supported using vertical piles and moving only in heave motion, when adding a fixed submerged horizontal solid plate beneath it under different characteristics of regular waves.

\section{METHODOLOGY}

\subsection{Experimental Equipment}

The experimental tests were conducted in the flume of the Hydraulic Laboratory, faculty of engineering, Port Said University. The flume is made up of glass sheets with $1 \mathrm{~cm}$ thickness and has a total length $13 \mathrm{~m}$, clear width $0.3 \mathrm{~m}$, and wall height $0.5 \mathrm{~m}$. All tests had been carried out at a constant water depth of $0.3 \mathrm{~m}$. The used flap type wave generator was able to generate regular waves with 0.6 to 1 second periods and 4 to $12 \mathrm{~cm}$ high, respectively. At the end of the flume, a wave absorber, made up from well graded gravels having a gradual slope, was installed to prevent wave's reflection. The water surface level was measured using four HR Wallingford wave gauges installed around the tested model which have accuracy of $0.1 \mathrm{~mm}$ and sample frequency was up to $100 \mathrm{~Hz}$.

Three wave gauges were installed at the wave generator side for measuring time series water surface motion and separating incident and reflective waves, while another was used at the absorber side for gauging the transmitted waves. "A 16 bit A/D" converter attached to PC had been used for converting analogue signals to digital data. The time series data of the waves were collected by the same computer, "HR WAVE DATA" software package was used to carry out the reflection analysis. All the necessary laboratory investigations including gauges calibration, wave decay and friction had been taken into considerations. Figure 1 shows the flume and the used equipment from an overall view.

\subsection{Models properties and test conditions}

Three different models had been considered in this study. The first model tested (Model A), is the submerged fixed horizontal plate breakwater SFHPB. The plate which made up of acrylic fibers sheets, with constant width of $29 \mathrm{~cm}$ normal to the flume and constant length of $25 \mathrm{~cm}$ in the direction of flume and waves, had been fastened to four rounded aluminum piles. The SFHPB had been tested for three different plate thicknesses, $t_{p}=6,12$ and $24 \mathrm{~mm}$ under two different submergence depths, $d_{p}=10$ and $15 \mathrm{~cm}$, respectively. The second model tested (Model $\mathrm{B}$ ), is the stepped-slope floating breakwater SSFB which made up the same material of the plate with constant thickness 6 mm thick and has the same width of model A but different length $=24 \mathrm{~cm}$, had been supported to the rounded aluminum piles through four rollers. This system allowed model B to slide and move only in heave with very limited roll motion. The SSFB had been tested under four different drafts of $D_{f}=5.0,6.0,7.5$ and 9.5 $\mathrm{cm}$, respectively in which drafts had been adjusted by placing weights inside model. The third model tested (Model C), is the composite stepped-slope floating breakwater CSSFB consists of the composition of Model (A) and Model (B) to produce the composite steppedslope floating breakwater with submerged fixed horizontal plate. This composite breakwater had been tested using all parameter mention for both models A and B. All tests had been carried out for constant water depth of $d=0.3 \mathrm{~m}$. Geometrical characteristics of different models are illustrated in Figure2.

It had been noticed that contacts occurred between the floating body during its heave motion and the submerged fixed plate. For these recorded condition, the transmission and reflection coefficients have been excluded from the introduced results.

\subsection{Performance Evaluation Method for Models}

Before each experiment carried out for the tested models, all the necessary laboratory considerations had been made including, check of water depth, proper test period, gauges calibrations, validity of the absorber and all other important factors necessary for the experimental work. For each experiment, four time series wave signals had been recorded. The three wave signals recorded in front of the model (wave generator side) represent the composite wave consists of the incident and the reflected waves at three different positions. 


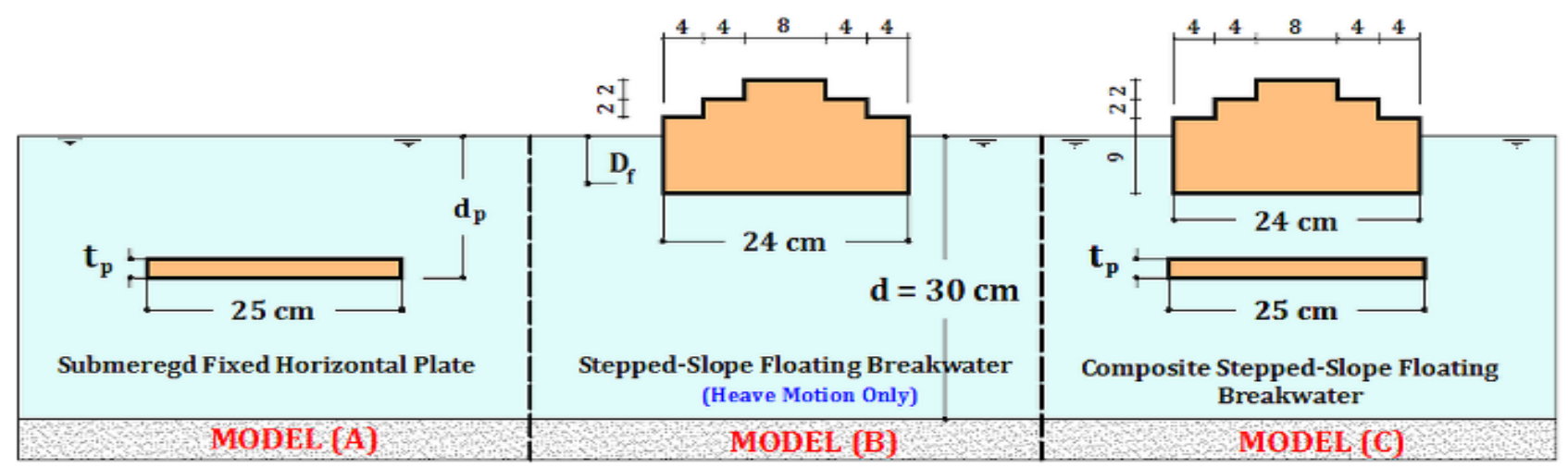

Figure 2: Characteristics of various tested breakwater models.

The recorded three wave signals in front of the model were used in order to decompose the composite wave into the incident wave height, $H_{i}$ and the reflected wave height, $H_{r}$ using 'HR WAVE DATA' software package. While, the fourth recorded wave signal represents the transmitted waves behind the model (absorber side) was used to determine the transmitted wave height, $H_{t}$. Thus, the performance of each tested model could be expressed in the form of reflection coefficient, $C_{r}=H_{r} / H_{i}$ and transmission coefficient $C_{t}=H_{t} / H_{i}$. Knowing the values of $C_{r}$ and $C_{t}$ for each experiment, the loss coefficient which represents wave energy loss, $C_{l}$ could be calculated from equation (1).

$C_{t}^{2}+C_{r}^{2}+C_{l}^{2}=1$ equation (1)

\section{Results and Discussion}

The results obtained for the three tested models will be discussed and analyzed here. The hydrodynamic performance of each tested model is represented in the form of $C_{t}, C_{r}$ and $C_{l}$ versus $B / L$ for a range of wave steepness $H_{i} / L$ of 0.026 to 0.114 .

\subsection{Submerged Fixed Horizontal Plate Breakwater (SFHPB)}

In the first group of experiments, the transmission coefficient results of the SFHPB (Model A) had been compared to these obtained by Tolba and Balah [20], as shown in figure 3 , for plate relative submergence depth $d_{p} / d=0.33$ and different plate relative thicknesses ratios, $t_{p} / d=2,4$ and $8 \%$ respectively. The figure shows that $C_{t}$ values measured in the present work are very close to range of the results introduced by Tolba and Balah [20] which is a good indication of the validity of the measurement method used in the present work.

The full hydrodynamic performance of the SFHPB (Model A) represented with the values of $C_{t}, C_{r}$ and $C_{l}$ versus $B / L$ is given in figures (4.a), (4.b) and (4.c) respectively. Figures (4.a) and (4.c) clarify that, for the tested range of $B / L$, the best performance of the tested system associated with averaged minimum transmission coefficient, $C_{t}=0.637$ and averaged maximum loss coefficient, $C_{l}=0.73$ of the SFHPB occurs with system configuration of $d_{p} / d=0.33$ and $t_{p} / d=2 \%$. While, the lowest performance of the same tested system with averaged maximum transmission coefficient, $C_{t}=0.754$

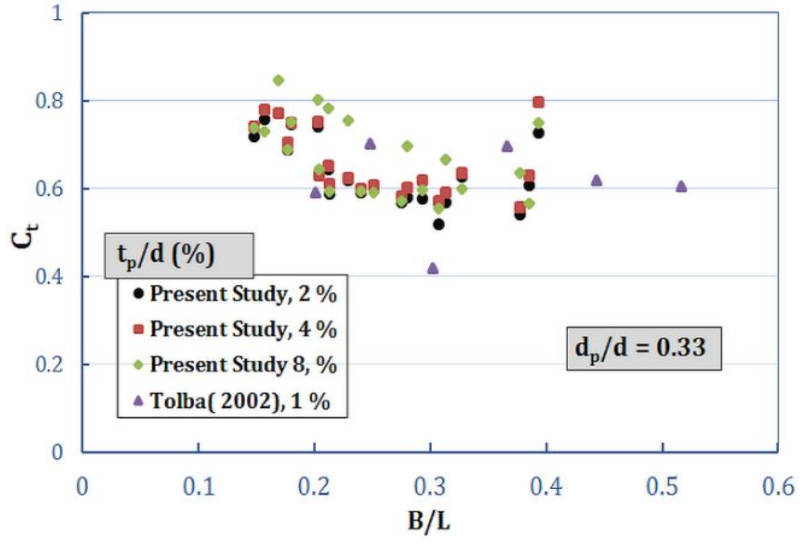

Figure 3: Comparison between $C_{t}$ results for the submerged horizontal plate.

and minimum loss coefficient, $C_{l}=0.603$ occurs with system configuration of $d_{p} / d=0.50$ and, $t_{p} / d=2 \%$. This previous findings prove that $d_{p} / d$ has significant effect on the performance of the SFHPB.

On the other hand, for the same $d_{p} / d$ ratio and $B / L$ range of the tested system, the results showed that the averaged $C_{t}, C_{r}$ and $C_{l}$ values varies in narrow range with $t_{p} / d$ as shown in figures (4.a) to (4.c). For example, for $d_{p} / d=0.33$, the averaged values of $C_{t}$ are 0.637 , 0.656 and 0.673 with corresponding $C_{r}$ values of 0.202 , 0.201 and 0.235 for $t_{p} / d=2,4$ and $8 \%$ respectively. While, for $d_{p} / d=0.50$, the averaged values of $C_{t}$ are $0.754,0.727$ and 0.724 with corresponding $C_{r}$ values of $0.19,0.14$ and 0.13 for $t_{p} / d=2,4$ and $8 \%$ respectively.

\subsection{Stepped-Slope Floating Breakwater (SSFB)}

In the second group of experiments, the hydrodynamic performance of the SSFB (Model B), had been investigated and represented with the values of $C_{t}$, $C_{r}$ and $C_{l}$ versus $B / L$ as shown in figures (5.a), (5.b) and (5.c) respectively. The model had been tested under four different drafts $D_{f}=5,6,7.5$ and $9.5 \mathrm{~cm}$ with relative drafts, $D_{f} / d=0.167,0.2,0.25$ and 0.317 respectively for the same range of $B / L$ used for model A. Figure (5.a) shows that the transmission coefficient, $C_{t}$ decreases with the increase of $B / L$ ratio and that $C_{t}$ also decreases with the increase of the relative drafts $D_{f} / d$. 

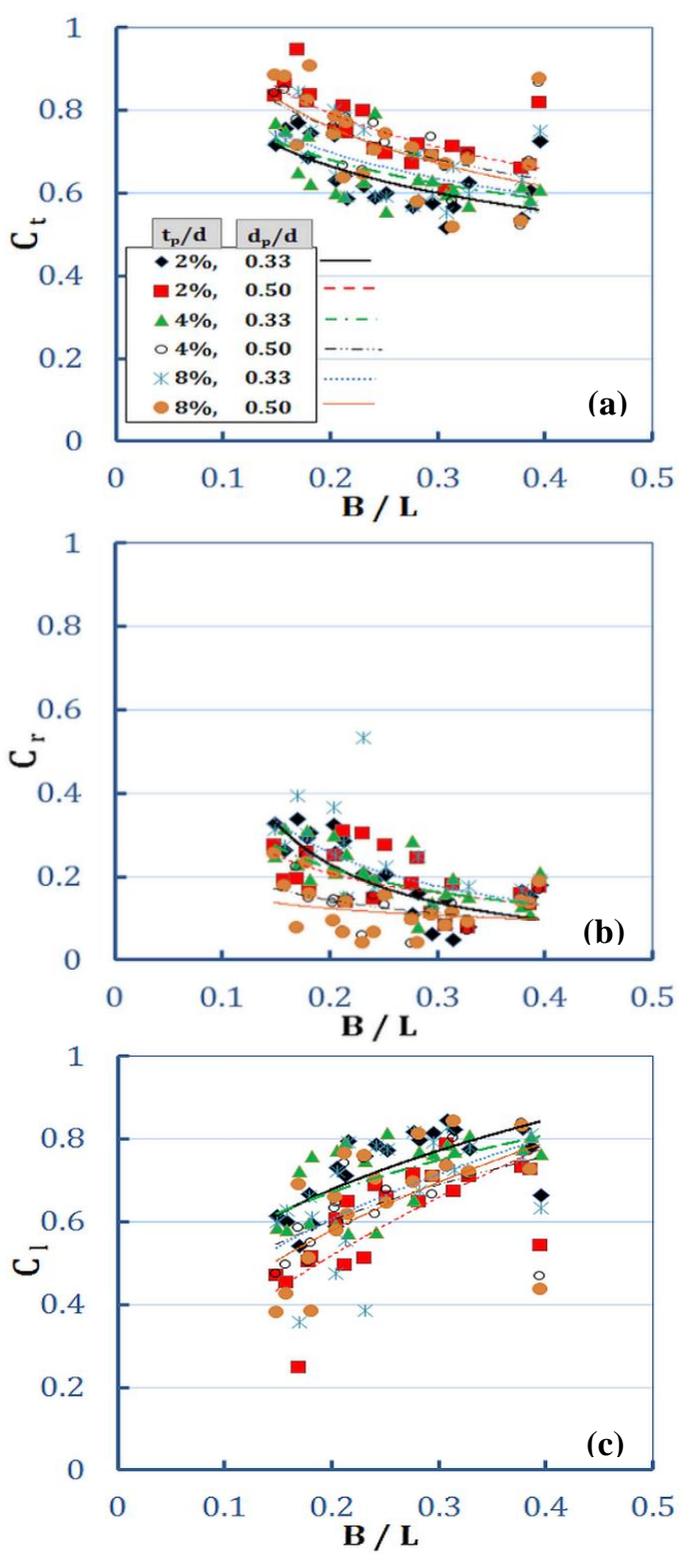

Figure 4: Hydrodynamic performance of Submerged fixed horizontal Plate Breakwater (SFHPB).

It was found that the averaged $C_{t}$ values of Model $\mathrm{B}=$ $0.35,0.3,0.28$ and 0.25 for $D_{f} / d=0.167,0.2,0.25$ and 0.317 , respectively.

\subsection{Composite Stepped-Slope Floating Breakwater (CSSFB)}

In the third group of experiments, the performance of the CSSFB (Model C) had been investigated and represented with the values of $C_{t}, C_{r}$ and $C_{l}$ versus $B / L$. The composite breakwater consists of the combinations of both models A and B.

Results of proposed composite stepped-slope Floating Breakwater (CSSFB) showed a good improvement in behavior of breakwater in damping incident wave and to discuss to what extend the improvement of results are, we will review result of one case of structures studied experimentally in this investigations, Figures (6.a), (6.b) and (6.c) show curves of $C_{t} C_{r}$ and $C_{l}$ respectively for composite model with a configuration of relative draft of
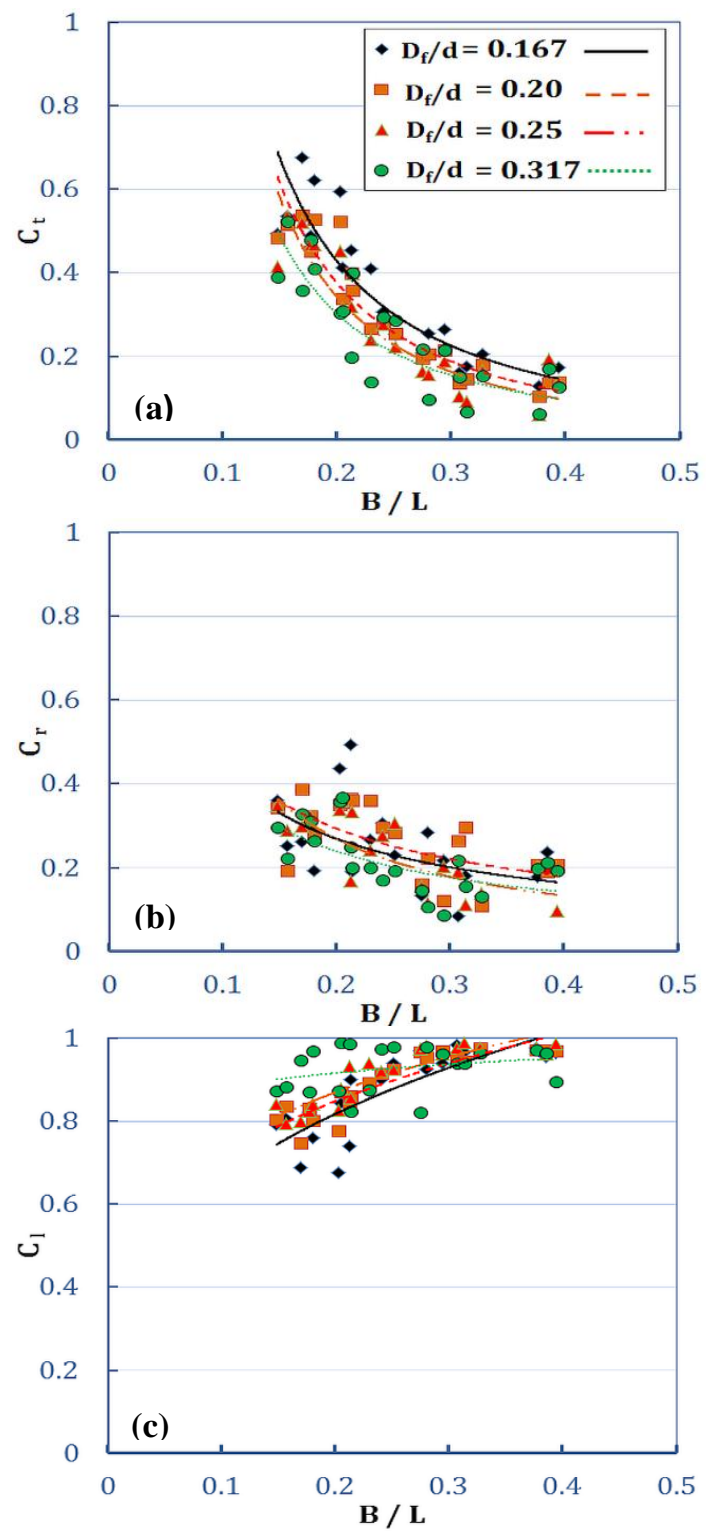

Figure 5: Hydrodynamic performance of SteppedSlope Floating Breakwater (SSFB).

the SSFB, $D_{f} / d=0.20$, plate relative thickness $t_{p} / d=2$ $\%$ and plate relative submergence depth $d p / d=0.33$. For the tested range of $B / L$, It is clear from figure (6.a) that $C_{t}$ values ranges between 0.72 to 0.57 for the SFHPB (Model A), 0.63 to 0.12 for the SSFB (Model B) and 0.4 to 0.06 for CSSFB (Model C).

These results indicate that the hydrodynamic performance of the stepped-slope floating breakwater SSFB had been improved with significant value as its $C_{t}$ values showed average reduction of $44 \%$ when adding submerged fixed horizontal plate SFHP underneath the floating body. Figure (6.b) assures the previous findings as the averaged values of reflection coefficient, $C_{r}$ of the composite model had been improved by $7 \%$, the coefficient of energy loss, $C_{l}$ has the greater values for 
(Model C) followed by (Model B) and then (Model A) as shown in figure (6.c).
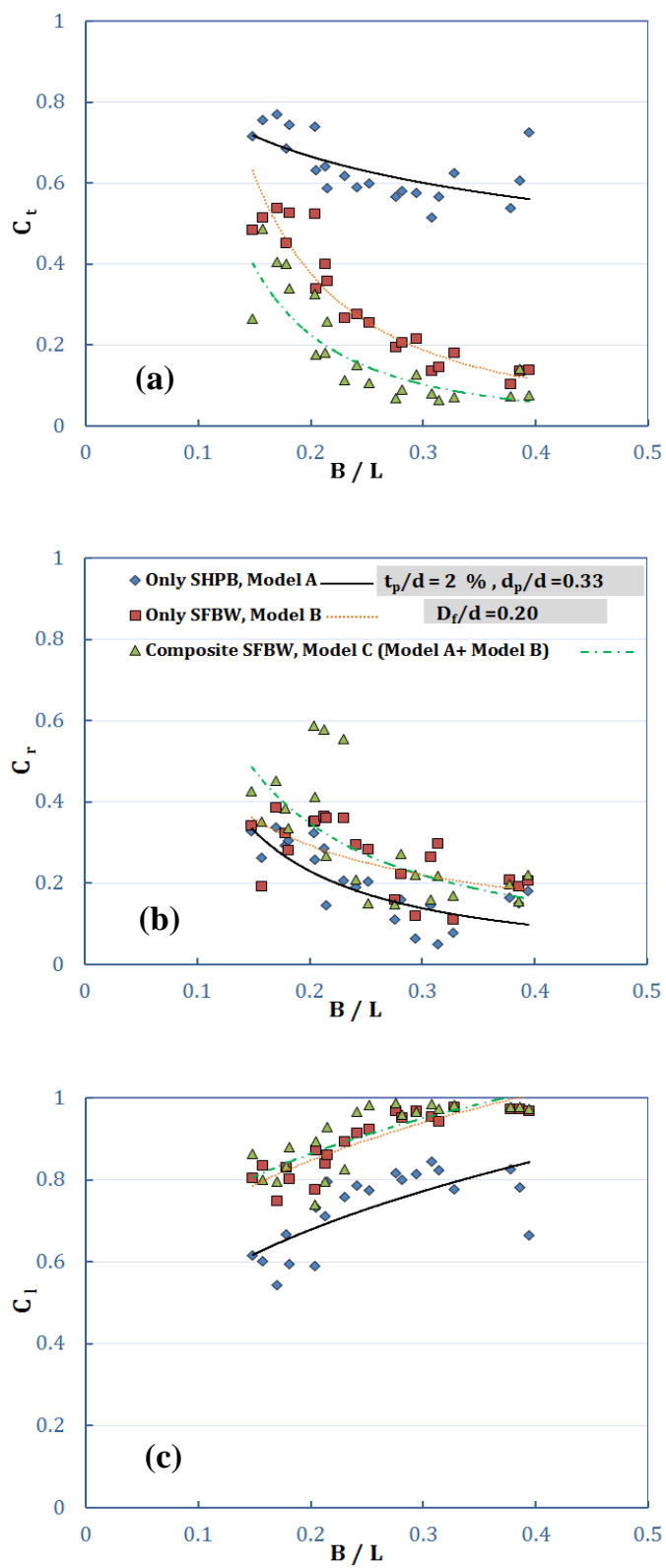

Figure 6: hydrodynamic performance of Composite Stepped-Slope Floating Breakwater (CSSFB). $\left(D_{f} / d=0.20, d_{p} / d=0.33, t_{p} / d=2 \%\right)$

\subsubsection{Effect of plate relative submergence depth}

Figures (7.a), (7.b) and (7.c) show an example for the performance of the composite model with a configuration of relative draft of the SSFB, $D_{f} / d=0.167$, plate relative thickness $t_{p} / d=4 \%$ and plate relative submergence depth $d_{p} / d=0.33$. For the tested range of $B / L$, It is clear from figure (7.a) that $C_{t}$ values ranges between 0.73 to 0.59 for the SFHPB (Model A), 0.69 to
0.15 for the SSFB (Model B) and 0.28 to 0.08 for CSSFB (Model C).

These results indicate that the hydrodynamic performance of the stepped-slope floating breakwater SSFB had been improved with significant value as its $C_{t}$ values showed reduction of $52 \%$ when adding submerged fixed horizontal plate SFHP underneath the floating body. Figure (7.b) assures the previous findings as the averaged values of reflection coefficient, $C_{r}$ of the composite model had been improved by $18 \%$ and became higher than that measured for model $\mathrm{B}$ for the same tested range of $\mathrm{B} / \mathrm{L}$ and as a result, the coefficient of energy loss, $C_{l}$ has the greater values for (Model $\mathrm{C}$ ) followed by (Model B) and then (Model A) as shown in figure (7.c).
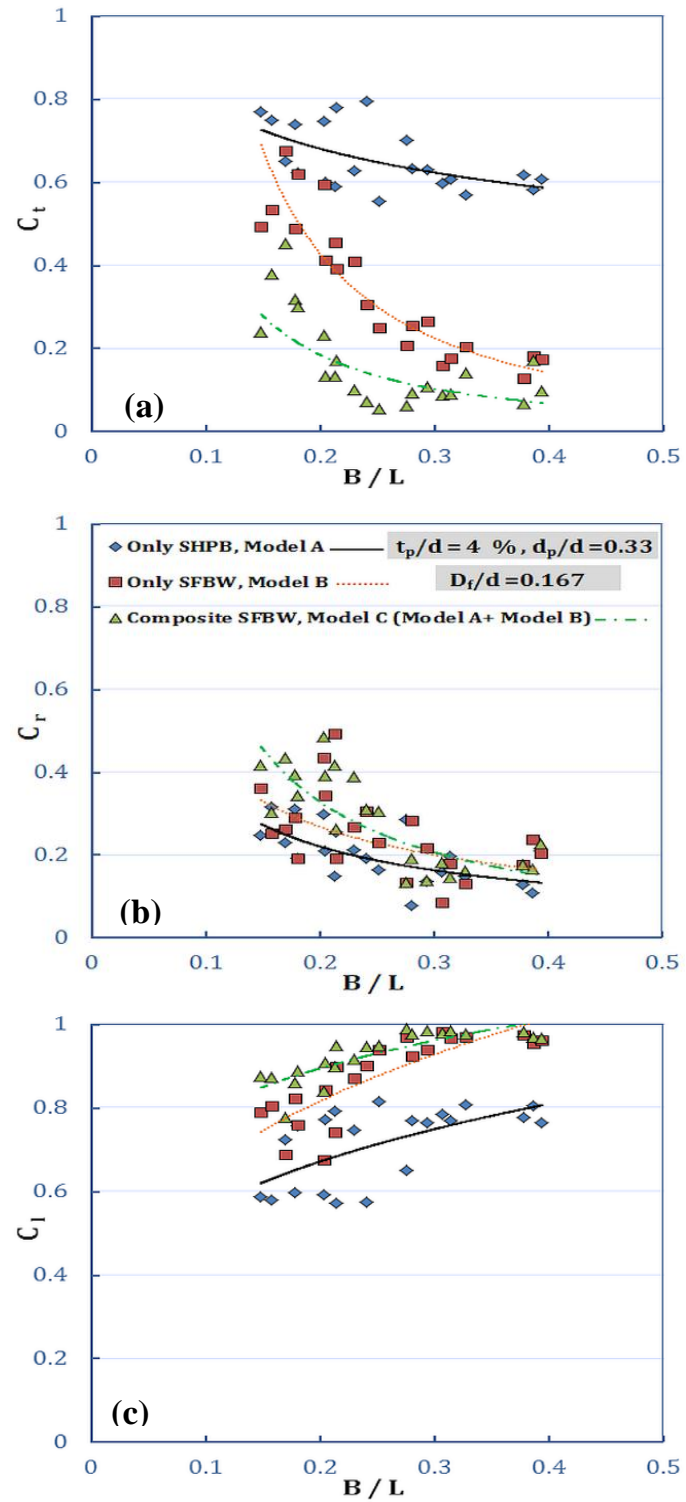

Figure 7: Effect of plate relative submergence depth on the hydrodynamic performance of Composite Stepped-Slope Floating Breakwater (CSSFB). $\left(D_{f} / d=0.167, d_{p} / d=0.33, t_{p} / d=4 \%\right)$ 
Figures 8 (a,b and c) represents the performance of the composite model with another configuration of floating body relative draft of the SSFB, $D_{f} / d=0.167$, plate relative thickness $t_{p} / d=4 \%$ but with plate relative submergence depth $d_{p} / d=0.50$. Figure (8.a) shows that for the same tested range of $B / L, C_{t}$ values ranges between 0.84 to 0.59 for the SFHPB (Model A), 0.7 to 0.17 for the SSFB (Model B) and 0.62 to 0.09 for the CSSFB (Model C). These results indicate that the hydrodynamic performance of this stepped-slope floating breakwater SSFB had been improved too, but with lower values than that obtained when using $d_{p} / d=$ 0.33 , as its $C_{t}$ values showed reduction of only $23 \%$ when adding submerged fixed horizontal plate SFHP underneath the floating body. Thus, it is clear that the submergence depth of the horizontal plate has significant influence on the efficiency of the composite breakwater.

This fact appears obviously in figure (8.b) as $C_{r}$ values of the composite model (Model C) decreased and became lower than that belong to the stepped-slope floating body only (Model B) due to the increase of the relative plate submergence depth ratio, $d_{p} / d$. the coefficient of energy loss, $C_{l}$ has the greater values for (Model C) followed by (Model B) and then (Model A) as shown in figure (8.c).
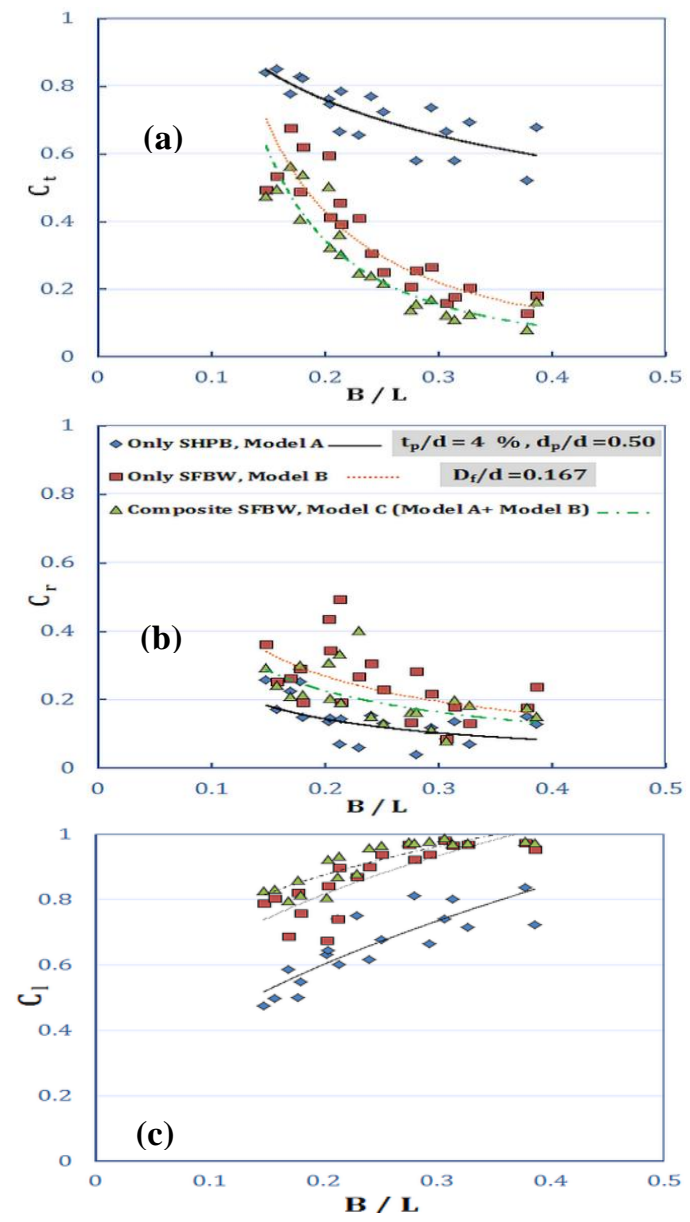

Figure 8: Effect of plate relative submergence depth on the hydrodynamic performance of CompositeStepped-Slope Floating Breakwater (CSSFB). $\left(D_{f} / d=0.167, d_{p} / d=0.50, t_{p} / d=4 \%\right)$

\subsubsection{Effect of floating body relative draft}

Figures (9) (a, b, c, d, e, f) discuss the effect of floating body relative draft ratio, $D_{f} / d$ on the performance of the composite tested model (Model C). The figures illustrate the change of $C_{t}$ values versus $B / L$ when using different floating body relative draft ratio, $D_{f} / d=0.167,0.2,0.25$ and 0.317 for the two configurations of $d p / d$ ratio $=0.33$ and 0.50 and using different values of the ratio $t_{p} d$.

Firstly we should mention here that results of $D_{f} / d=$ 0.317 and some results of $D_{f} / d=0.25$ and 0.20 models were excluded in some configurations of $d p / d$ ratio $=$ 0.33 , because of the contact noticed in some experiments between the floating body and the submerged fixed plate during its heave motion. Furthermore models which excluded some points displayed in a point values form not in a curve form.

Figure (9.a) illustrates $C_{t}$ values for the composite model (Model C) with constant ratio, $t_{p} / d=2 \%$, three different ratios of $D_{f} / d=0.167,0.2$ and 0.25 at constant ratio of $d_{p} / d=0.33$. The results show that the best performance of the composite model occurs when $D_{f} / d=$ 0.25 with a maximum value of $C_{t}=0.1$ for the allowable tested range of $B / L$. While for $D_{f} / d=0,167$ and 0.20 , the results show that $C_{t}$ decrease with the increase of $B / L$ with better performance for the configuration using $D_{f} / d$ $=0.167$. In figure (9.b) with $t_{p} / d=4 \%$, the results show approximate values of $C_{t}$ for the common tested range of $B / L$. However, for whole range of $\mathrm{B} / \mathrm{L}$, results of models with $D_{f} / d=0.167$ and 0.20 , assured that $C_{t}$ decrease with the increase of $B / L$ but with better performance for the configuration using $D_{f} / d=0.20$.

Figure (9.c) shows results of transmission coefficients for structure configuration of $d p / d$ ratio $=0.33, t_{p} / d=$ $8 \%$ with models having $D_{f} / d=0.167$ and $D_{f} / d=0.2$, we should mentioned here that models of $D_{f} / d=0.25$ and 0.317 , in additional to some results of model with $D_{f} / d=$ 0.20 had been excluded, due to the reason mentioned before. The tested system showed significant changes on its behavior as the results showed remarkable improvement in its wave attenuation performance and $C_{t}$ values decreased obviously when using $D_{f} / d=0.167$ for long crested waves of smaller values of $\mathrm{B} / \mathrm{L}$, while conversely for the maximum values of $\mathrm{B} / \mathrm{L}$.

Figure (9.d) illustrates $C_{t}$ values for the composite model (Model C) with constant ratio, $t_{p} / d=2 \%$, four different ratios of $D_{f} / d=0.167,0.2,0.25$ and 0.317 at constant ratio of $d_{p} / d=0.50$. Results show a great matching between performance of the $D_{f} / d=0.167$ and 0.2 models with an average value of $C_{t}=0.3$, while the remaining two models show moderate matching between results with a supremacy in $D_{f} / d=0.25$ model efficiency having an average $C_{t}$ of 0.2 .

Figure (9.e) displays $C_{t}$ values for the composite model (Model C) with constant ratio, $t_{p} / d=4 \%$, four different ratios of $D_{f} / d=0.167,0.2,0.25$ and 0.317 at constant ratio of $d_{p} / d=0.50$. Previous results are 
repeated but in a smaller matching trending. Figure show
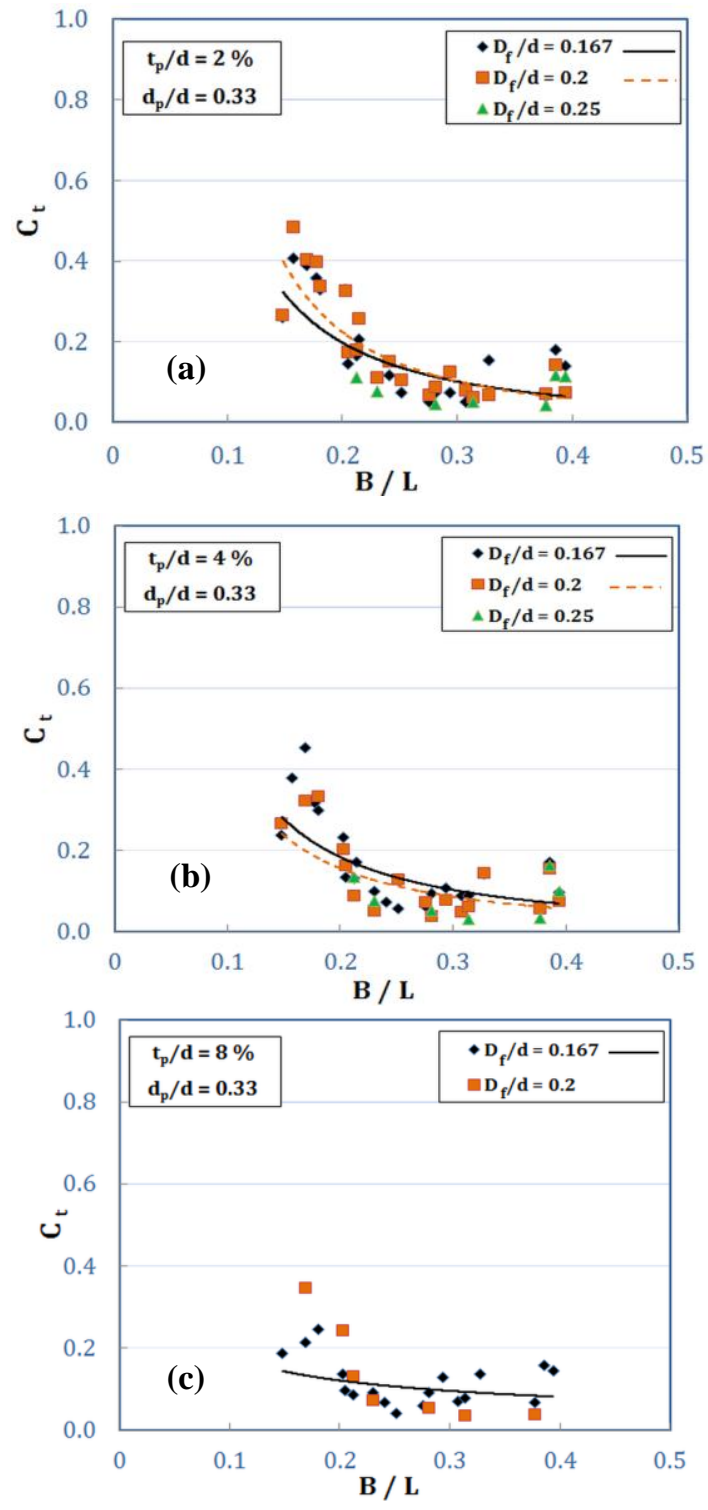

less values of $\mathrm{C}_{\mathrm{t}}$ is corresponding to $D_{f} / d=0.316$
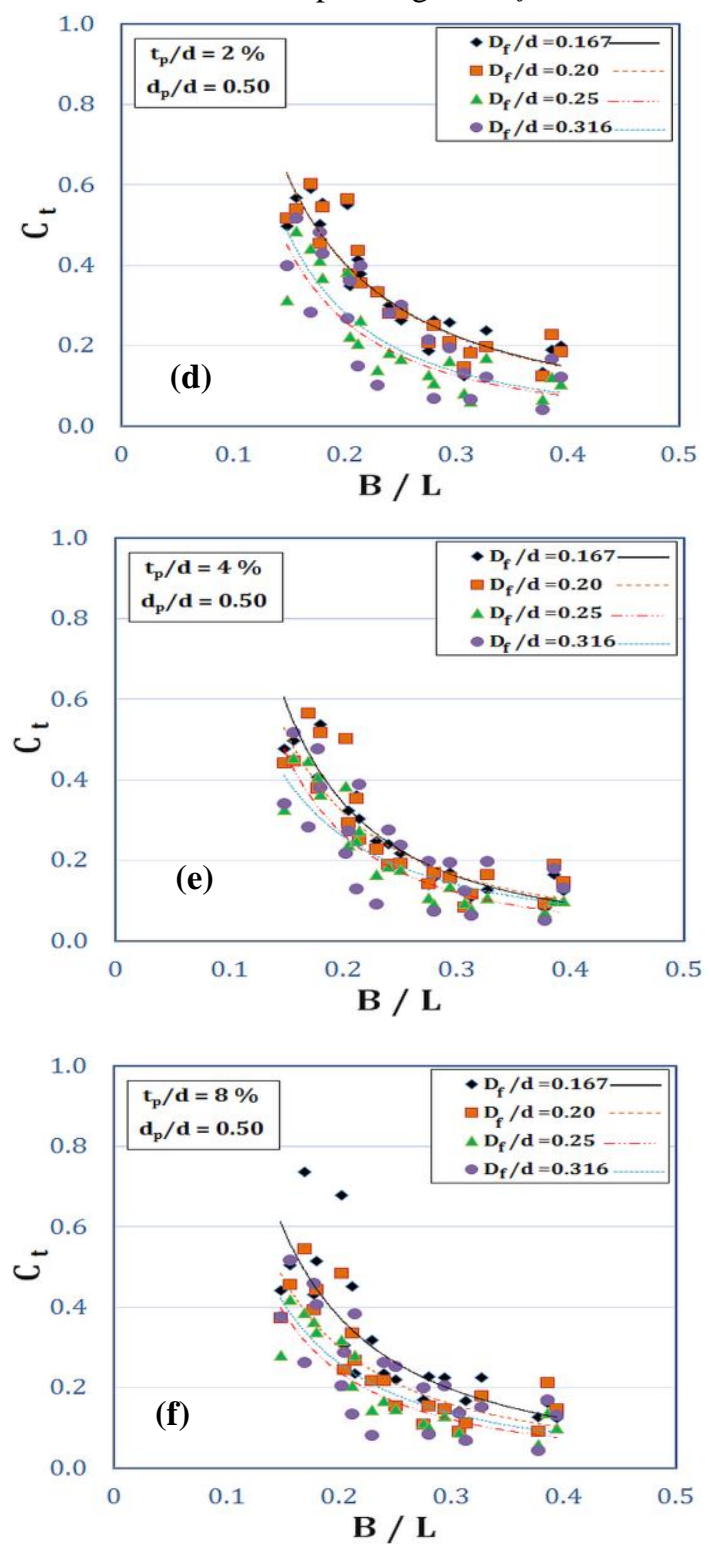

Figure 9: Effect of floating body relative draft on the hydrodynamic performance of Composite Stepped-Slope Floating Breakwater (CSSFB) for several configurations

model for a long crested waves with an average value of 0.3 , while $D_{\mathrm{f}} / d=0.25$ model possessed the minimum values of ct with an average value of 0.12 .

Figure (9.f) illustrates $C_{t}$ values for the composite model (Model C) with constant ratio, $t_{p} / d=8 \%$, four different ratios of $D_{f} / d=0.167,0.2,0.25$ and 0.317 at constant ratio of $d_{p} / d=0.50$. Results show that the deeper of draft distance of the model mean the less values of $\mathrm{Ct}$, curves show also a similar between results of $D_{f} / d=0.25$ and 0.317 models but priority results of $\mathrm{Ct}$ are the results of $D_{f} / d=0.25$ model with an average value of 0.19 .

\subsubsection{Effect of plate relative thickness}

Figures 10 (a, b, c and d) discuss the effect of the plate relative thickness, $t_{p} / d$ on the performance of the composite tested model (Model C) at constant plate relative submergence depth $d_{p} / d=0.50$ and four different floating body relative draft ratios, $D_{f} / d$. The figures indicate that, at $d_{p} / d=0.50$, the effect of plate thickness appears in the configurations using $D_{f} / d=$ 0.167 and 0.2 as shown in figures 10 ( $\mathrm{a}$ and $\mathrm{b}$ ). On the other hand, figures 10 (c and d) show no influence of the different plate thicknesses on the transmission coefficients, $C_{t}$ for the configurations using $D_{f} / d=0.25$ and 0.317 except these small differences noted at the lower values of $\mathrm{B} / \mathrm{L}$. 


\section{CONCLUSIONS}

A submerged horizontal fixed plate had been used under the pile supported stepped-slope floating breakwater type for the purpose of investigating its hydrodynamic performance improvement. The resulted composite breakwater had been investigated experimentally under different plate submergence depths, plate thicknesses and different drafts of the stepped-slope body. The experiments carried out on the plate as single attenuator showed attenuation capability when located at relative submergence depth, $d_{p} / d=0.33$ higher than obtained for $d_{p} / d=0.50$. Experiments operated on the stepped-slope floating breakwater illustrate that increasing of relative draft of breakwater give higher performance as a wave attenuator. Experiments also showed that stepped-slope floating breakwater recorded significant improvement on its hydrodynamic performance when using the submerged plate underneath the floating body. The improvement of its hydrodynamic performance recorded up to $52 \%$ and $23 \%$ reduction in transmission coefficients when installing the plate underneath the floating body at relative submergence depths, $d_{p} / d=0.33$ and 0.5 respectively. For relative submergence depth $d_{p} / d=$ 0.33 , the experiments showed some contacts between the bottom of the floating body during its movement in heave and the plate specially in the cases of floating body relative draft of 0.25 and 0.317 while, no contacts recorded for $d_{p} / d=0.50$.

The discussion of results for influence of relative draft of the floating stepped-slope breakwater in the composite breakwater structure showed that model of $D_{f} / d=0.25$ possessed the least values of transmission coefficient in comparison with other relative drafts, If it is possible to be used.

The thickness of the plate showed also an effect on the performance of the tested system, which appears in both configurations when using $d_{p} / d=0.33$ and 0.5 especially for small relative drafts of the floating body. This effect tends to decrease with the increase of plate submergence depth.

\section{REFERENCES}

[1]. Aghili et al., (2014), "Simulating the interaction of solitary wave and submerged horizontal plate using SPH method", International Journal of Physical Research, 2 (2), pp 16-26.

[2]. Chen, Z. et al., (2012), "Time-domain hydrodynamic analysis of pontoon-plate floating breakwater", Water Science and Engineering, 5(3), pp 291-303, doi:10.3882/j.issn.16742370.2012.03.005.
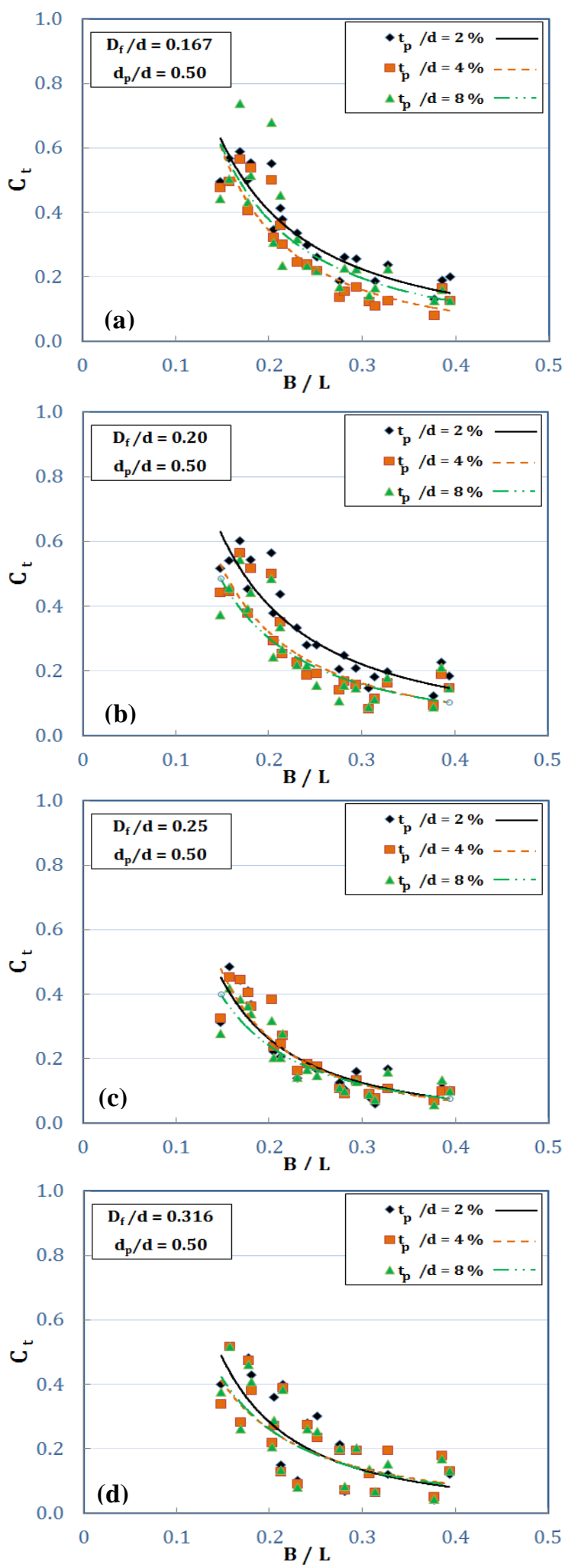

Figure 10: Effect of plate relative thickness on the hydrodynamic performance of Composite SteppedSlope Floating Breakwater $(\mathrm{CSSFB}),\left(d_{p} / d=\mathbf{0 . 5 0}\right)$ 
[3]. Dauer, L., (1984), "Energy transport with a vertical immersed wall and a horizontal plate", diploma thesis, Institute of Hydraulic Engineering and Water Management, Technical University, Germany.

[4]. Drieman, R., (2011), "Feasibility study on the use of a floating breakwater to protect a new artificial beach in Balchik", Master of Civil Engineering, Delft University of Technology, Netherlands.

[5]. Fousert, M.W., (2006), "Floating Breakwater, A Theoretical study of a dynamic wave attenuating system", Master of Civil Engineering, Delft University of Technology, Netherlands.

[6]. Graw, K.U., (1995), "Estimation of CtCoefficient for the submerged horizontal plate”, Proceeding XXVIAHR Congress, UK, Vol. 3, pp 245-250, London.

[7]. Heng, L. C., (2006), "system performance of a composite stepped-slope floating breakwater", Master's thesis, Faculty of Civil Engineering, University Technology, Malaysia.

[8]. Koftis, T., and Prinos. P., (2005), “improved hydrodynamic efficiency of pontoon -type floating breakwaters", XXXI IAHR congress, pp 40474056, Seoul, Korea.

[9]. Koftis, T.H.,/ Prinos, P.,/ Koutandos, E., (2006), " $2 D-V$ hydrodynamics of wave-floating breakwater interaction", Journal of Hydraulic Research Vol. 44, No. 4, pp. 451-469.

[10]. Koutandos, E.V.,/ Karambas, Th.V.,/ Koutitas, C.G.,/ Prinos, P.E., (2002), "Floating Breakwaters Efficiency in Intermediate and Shallow Water", International Conference on Hydro-Science and Engineering's, Warsaw, Poland.

[11]. Koutandos, E.,/ Karambas, T.,/ Koutitas, C., (2004). "Floating Breakwater Response to Waves Action Using a Boussinesq Model Coupled with a 2DV Elliptic Solver", Journal of Waterway Port, Coastal and Ocean Engineering, doi.10.1061/(ASCE)0733950X(2004)130:5(243), 243-255.

[12]. Koutandos, E.,/Prinos, P.,/ Gironella, X., (2005), "Floating breakwaters under regular and irregular wave forcing: reflection and transmission characteristics", Journal of Hydraulic Research Vol. 43, No. 2 (2005), pp. 174-188.
[13]. Koutandos, E.V., and Prinos, P.E., (2011), "Hydrodynamic characteristics of semiimmersed breakwater with an attached porous plate”, Ocean Engineering 38, 34-48.

[14]. Kolahdoozan, M.,/ Taheri O.,/ Ghasemi H., (2015), "Hydrodynamic Performance Evaluation of Step Floating Breakwaters through Experiment and Artificial Intelligence", Proceedings of the International Conference on Civil, Structural and Transportation Engineering Ottawa, Ontario, May 4 - 5, 2015 Paper No. 293, Canada.

[15]. Merlevede, K., (2012), "Study of the functional design of a floating offshore breakwater", Master of Civil Engineering, Ghent University, Belgium.

[16]. Patarapanich, M., and Cheong, H.F., (1989), "Reflection and transmission characteristics of regular and random waves from a submerged horizontal plate", Costal Engineering, 13, pp 161-182.

[17]. Teh, H., (2002). "Wave damping characteristics of a stepped-slope floating breakwater", Master's thesis, Faculty of Civil Engineering, University Technology Malaysia.

[18]. Teh, H., and Ismail, H., (2013), "Hydraulic Characteristics of a Stepped-slope Floating Breakwater", 4th International Conference on Energy and Environment, doi:10.1088/1755$1315 / 16 / 1 / 012060$

[19]. Tolba, E., (1999), "behavior of floating breakwaters under wave action”, Ph.D. Thesis, University of Wuppertal \& Suez Canal University.

[20]. Tolba, E.,/ Balah, M.I., (2002), "Transmission of Regular Waves Past Composite Vertical Barrier - Submerged Horizontal Plate Breakwater", Port Said Engineering Research Journal, Vol.6. (2), pp 430-441. 